\title{
Diabetes autoimune latente do adulto (LADA): fisiopatologia, critérios diagnósticos e associação com doenças autoimunes
}

\author{
Adult latent autoimmune diabetes (LADA): pathophysiology, diagnostic criteria and association \\ with autoimmune diseases
}

Diabetes autoinmune latente del adulto (LADA): fisiopatología, criterios diagnósticos y asociación con enfermedades autoinmunes

Bárbara Queiroz de Figueiredo ORCID: https://orcid.org/0000-0003-1630-4597 Centro Universitário de Patos de Minas, Brasil

E-mail: barbarafigueiredo@unipam.edu.br Gabriele Durante ORCID: https://orcid.org/0000-0001-6443-1123 Centro Universitário Atenas, Brasil E-mail: gabidurante8@gmail.com

Gardênia Silva Amorim

ORCID: https://orcid.org/0000-0001-7888-8562 Centro Universitário de Atenas, Brasil E-mail: gardênia.sa@hotmail.com

Isadora Queiroz Presot ORCID: https://orcid.org/0000-0003-1117-1211

Centro Universitário Euroamericano, Brasil E-mail: presotisadora@gmail.com

Júlia Rodrigues Souza

ORCID: https://orcid.org/0000-0002-7246-6366 Centro Universitário de Patos de Minas, Brasil E-mail: juliarsouza01@ gmail.com

Lavínya de Souza Soares ORCID: https://orcid.org/0000-0001-8214-905X Centro Universitário de Patos de Minas, Brasil

E-mail: lavinyass@unipam.edu.br

Nicolly Skarlet Souto Oliveira ORCID: https://orcid.org/0000-0003-3304-9946 Centro Universitário de Patos de Minas, Brasil E-mail: nicollyskarlet@gmail.com

Pedro Eduardo Pereira Assunção ORCID: https://orcid.org/0000-0001-9163-6271 Centro Universitário de Patos de Minas, Brasil E-mail: pedroevzt@gmail.com Sabrina Siqueira Porto ORCID: https://orcid.org/0000-0002-8256-6459 Centro Universitário de Patos de Minas, Brasil E-mail: sabrinaporto@ unipam.edu.br

Vinícius Leandro Oliveira Medeiros ORCID: https://orcid.org/0000-0003-1422-7452

Universidade Católica de Brasília, Brasil E-mail: vinyleandro.bsb@outlook.com

\begin{abstract}
Resumo
Introdução: trata-se de uma forma de diabetes em que a autoimunidade contra as células beta pancreáticas se desenvolve de maneira mais arrastada e a obrigatoriedade do uso da insulina pode se dar após vários de diagnóstico. No LADA, a disfunção da célula $\beta$ tem sido reportada como intermediária entre os dois principais tipos de diabetes mellitus. Objetivo: evidenciar responder a fisiopatologia, critérios diagnósticos do LADA e sua associação com doenças autoimunes. Metodologia: trata-se de uma pesquisa descritiva do tipo revisão integrativa da literatura. A pesquisa foi realizada através do acesso online nas bases de dados National Library of Medicine (PubMed MEDLINE), Scientific Electronic Library Online (Scielo), Google Scholar, Biblioteca Virtual em Saúde (BVS) e EBSCO Information Services. Resultados e discussão: por ser latente e de lenta progressividade, os critérios de diagnóstico de LADA ainda são muito confusos, levando a uma alta taxa de diagnósticos incorretos. Há evidências
\end{abstract}


em diabetes autoimune para um continuum de suscetibilidade genética, que se estende de um efeito marcante no diabetes tipo 1 de início na infância até o efeito relativamente limitado detectado em LADA. O diagnóstico de LADA é atualmente baseado em três critérios: idade adulta no início do diabetes, a presença de autoanticorpos de ilhotas circulantes; e falta de necessidade de insulina por pelo menos 6 meses após o diagnóstico. Conclusão: Diagnosticar o diabetes autoimune em indivíduos erroneamente classificados como tipo 2 é importante, pois alerta para provável deficiência de insulina, evitando o atraso na insulinoterapia.

Palavras-chave: Diabetes autoimune latente do adulto; Autoimunidade; Células beta-pancreáticas; Diagnóstico; Fisiopatologia.

\begin{abstract}
Introduction: this is a form of diabetes in which autoimmunity against pancreatic beta cells develops more slowly and the mandatory use of insulin may occur after several diagnoses. In LADA, $\beta$-cell dysfunction has been reported as intermediate between the two main types of diabetes mellitus. Objective: to demonstrate answering the pathophysiology, diagnostic criteria of LADA and its association with autoimmune diseases. Methodology: this is a descriptive research of the integrative literature review type. The search was carried out through online access in the National Library of Medicine (PubMed MEDLINE), Scientific Electronic Library Online (Scielo), Google Scholar, Virtual Health Library (VHL) and EBSCO Information Services databases. Results and discussion: because it is latent and slowly progressive, the diagnostic criteria for LADA are still very confusing, leading to a high rate of misdiagnosis. There is evidence in autoimmune diabetes for a continuum of genetic susceptibility, which extends from a marked effect in childhood-onset type 1 diabetes to the relatively limited effect detected in LADA. The diagnosis of LADA is currently based on three criteria: adult age at onset of diabetes, the presence of circulating islet autoantibodies; and lack of need for insulin for at least 6 months after diagnosis. Conclusion: Diagnosing autoimmune diabetes in individuals wrongly classified as type 2 is important, as it alerts to a probable insulin deficiency, avoiding delays in insulin therapy.
\end{abstract}

Keywords: Adult latent autoimmune diabetes; Autoimmunity; Pancreatic beta cells; Diagnosis; Pathophysiology.

\title{
Resumen
}

Introducción: se trata de una forma de diabetes en la que la autoinmunidad frente a las células beta pancreáticas se desarrolla más lentamente y el uso obligatorio de insulina puede ocurrir después de varios diagnósticos. En LADA, se ha informado que la disfunción de las células $\beta$ es intermedia entre los dos tipos principales de diabetes mellitus. Objetivo: demostrar responder a la fisiopatología, criterios diagnósticos de LADA y su asociación con enfermedades autoinmunes. Metodología: se trata de una investigación descriptiva del tipo revisión integradora de la literatura. La búsqueda se realizó a través del acceso en línea en las bases de datos de la Biblioteca Nacional de Medicina (PubMed MEDLINE), la Biblioteca Electrónica Científica en Línea (Scielo), Google Scholar, la Biblioteca Virtual de Salud (BVS) y los Servicios de Información de EBSCO. Resultados y discusión: debido a que es latente y de progresión lenta, los criterios diagnósticos de LADA siguen siendo muy confusos, lo que lleva a una alta tasa de diagnósticos erróneos. Existe evidencia en la diabetes autoinmune de un continuo de susceptibilidad genética, que se extiende desde un efecto marcado en la diabetes tipo 1 de inicio en la niñez hasta el efecto relativamente limitado detectado en LADA. El diagnóstico de LADA se basa actualmente en tres criterios: la edad adulta al inicio de la diabetes, la presencia de autoanticuerpos de los islotes circulantes; y falta de necesidad de insulina durante al menos 6 meses después del diagnóstico. Conclusión: El diagnóstico de diabetes autoinmune en individuos mal clasificados como tipo 2 es importante, ya que alerta de una probable deficiencia de insulina, evitando retrasos en la terapia con insulina.

Palabras clave: Diabetes autoinmune latente del adulto; Autoinmunidad; Células beta pancreáticas; Diagnóstico; Fisiopatología.

\section{Introdução}

O diabetes latente autoimune do adulto (LADA), por definição, acomete pessoas de 25 anos ou mais, mas pode incidir em qualquer idade fazendo com que, muitas vezes, o paciente seja diagnosticado como tendo diabetes mellitus tipo 2 (DM2). Trata-se de uma forma de diabetes em que a autoimunidade contra as células beta pancreáticas se desenvolve de maneira mais arrastada e a obrigatoriedade do uso da insulina pode se dar após vários de diagnóstico. No LADA, a disfunção da célula $\beta$ tem sido reportada como intermediária entre os dois principais tipos de diabetes mellitus (DM1 e DM2) (Calsolari et al. 2008).

Do ponto de vista fisiopatológico, o DM1 resulta da falta absoluta ou quase absoluta de insulina enquanto o DM2 ocorre por resistência à ação da insulina nos tecidos e graus variáveis de redução na produção do hormônio. Fenômenos genéticos, imunológicos e metabólicos parecem convergir com outros processos de doença condicionando a apoptose da célula $\beta$ e contribuindo para o aparecimento da LADA. De acordo com Jones, et al. (2021), sendo ela identificada com um grupo de 
características clínicas e genéticas intermediárias entre os dois tipos de diabetes, nota-se que esse fenômeno ainda não está claro, sendo propostas, pelo autor, três teorias: como um fenótipo mais brando que inicia-se em idades mais tardias e que são semelhantes ao DM2, mas que requer insulina; como uma síndrome em que as fisiopatologias do DM1 e DM2 estão presentes em cada paciente; como um grupo heterogêneo decorrente de dificuldades de classificação.

O diagnóstico de LADA é tipicamente baseado no achado de hiperglicemia, associado à constatação de que a causa seja devida à uma falha das ilhotas de Langherans em produzir insulina, presença de sintomas agudos, índice de massa corporal $<25 \mathrm{~kg} / \mathrm{m} 2$, história pessoal ou familiar de outras doenças autoimunes. A presença de, pelo menos, duas destas características clínicas justifica o pedido de anticorpos contra as células beta pancreáticas. Tem-se, atualmente, quatro tipos de anticorpos: anti-glutamato descarboxilase (GADA), anti-insulina (IAA), anticélulas beta (ICA) e antitransportador de zinco. No LADA, os anticorpos anti-insulina (IAA) são raros, enquanto os GADA são característicos, mas estes podem se tornar negativos ao longo do tempo. Indivíduos com título elevado de GADA ou com positividade para maior número de anticorpos, geralmente apresentam um índice de massa corporal (IMC) inferior, menor secreção endógena de insulina e progressão mais rápida para insulinoterapia. Assim, o título de GADA pode permitir identificar indivíduos que respondem pior à terapêutica com antidiabéticos orais e que apresentam maior risco de cetoacidose (Silva, 2007).

A detecção de um nível baixo de peptídeo-C e anticorpos contra as ilhotas de Langerhans reforçam e sustentam este diagnóstico. Uma diferenciação com o diabetes tipo 2 pode ser feita porque as pessoas com LADA tipicamente têm níveis baixos, embora às vezes moderados, de peptídeo $\mathrm{C}$ à medida que a doença progride, e aqueles com resistência à insulina ou diabetes tipo 2 são mais propensos a ter níveis elevados de peptídeo $\mathrm{C}$ devido a uma produção excessiva de insulina. Clinicamente, os indivíduos com LADA representam um grupo heterogêneo de doentes com títulos variáveis de anticorpos, índice de massa corporal (IMC) e progressão para insulinoterapia (Couri, et al. 2009). Desse modo, o objetivo deste estudo é evidenciar responder a fisiopatologia, critérios diagnósticos do LADA e sua associação com doenças autoimunes.

\section{Metodologia}

Trata-se de uma pesquisa descritiva do tipo revisão integrativa da literatura, que buscou responder a fisiopatologia, critérios diagnósticos do LADA e sua associação com doenças autoimunes. A pesquisa foi realizada através do acesso online nas bases de dados National Library of Medicine (PubMed MEDLINE), Scientific Electronic Library Online (Scielo), Cochrane Database of Systematic Reviews (CDSR), Google Scholar, Biblioteca Virtual em Saúde (BVS) e EBSCO Information Services, no mês de agosto de 2021. Para a busca das obras foram utilizadas as palavras-chaves presentes nos descritores em Ciências da Saúde (DeCS): em inglês: "autoimmune diabetes", "LADA", "physiopathology", "clinical", "autoimmunity", em português: "diabetes autoimune", "LADA", "fisiopatologia", "clínica", "autoimunidade" e em espanhol: "diabetes autoinmune", "LADA", "fisiopatología", "clínica", "autoinmunidad".

Como critérios de inclusão, foram considerados artigos originais, que abordassem o tema pesquisado e permitissem acesso integral ao conteúdo do estudo, publicados no período de 2002 a 2021, em inglês, português e espanhol. O critério de exclusão foi imposto naqueles trabalhos que não estavam em inglês, português e espanhol, que não tinham passado por processo de Peer-View e que não elucidassem a fisiopatologia, critérios diagnósticos do LADA e sua associação com doenças autoimunes. A estratégia de seleção dos artigos seguiu as seguintes etapas: busca nas bases de dados selecionadas; leitura dos títulos de todos os artigos encontrados e exclusão daqueles que não abordavam o assunto; leitura crítica dos resumos dos artigos e leitura na íntegra dos artigos selecionados nas etapas anteriores. Assim, totalizaram-se 22 artigos científicos para a revisão integrativa da literatura, com os descritores apresentados acima, dos últimos dezenove anos e em línguas portuguesa, inglesa e espanhola. 


\section{Resultados e Discussão}

O conceito de diabetes autoimune latente do adulto, cuja sigla em inglês é LADA, de latent autoimmune diabetes in adults foi introduzido pela primeira vez em 1993, por Tuomi e Zimmet para definir pacientes diabéticos adultos que não requeriam insulina para controle da hiperglicemia nas fases iniciais da patologia, mas apresentavam autoanticorpos contra proteínas das células-beta e progressão mais rápida para necessidade de insulina. Trata-se de uma forma de diabetes em que a autoimunidade contra as células beta pancreáticas se desenvolve de maneira mais arrastada e a obrigatoriedade do uso da insulina pode se dar após vários de diagnóstico. No LADA, a disfunção da célula $\beta$ tem sido reportada como intermediária entre os dois principais tipos de diabetes (DM1 e DM2).

\section{- Fisiopatologia}

O diabetes autoimune latente em adultos (LADA) é um subtipo de diabetes autoimune de tipo 1. Por ser latente e de lenta progressividade, os critérios de diagnóstico de LADA ainda são muito confusos, levando a uma alta taxa de diagnósticos incorretos. A epidemiologia do diabetes autoimune, incluindo o diabetes tipo 1, é influenciada pela suscetibilidade genética, que modifica a idade no início. Há evidências em diabetes autoimune para um continuum de suscetibilidade genética, que se estende de um efeito marcante no diabetes tipo 1 de início na infância até o efeito relativamente limitado detectado em LADA (Liao et al. 2012).

Segundo estudo de Leslie et al. (2006), a análise de sobrevivência estima que gêmeos idênticos não diabéticos de probandos diagnosticados com diabetes tipo 1 com menos de 25 anos de idade têm 38\% de probabilidade de desenvolver diabetes, em comparação com apenas 6\% para gêmeos de probandos diagnosticados posteriormente (Hyttinen et al. 2003). Essa concordância de gêmeos notavelmente baixa para diabetes tipo 1 de início na idade adulta implica que o impacto genético no diabetes de início na idade adulta é limitado favorecendo um impacto substancial de fatores ambientais. Há um continuum relacionado à idade na diminuição da concordância de gêmeos, sugerindo que o declínio na influência genética é gradual, consistente com uma influência em fases em uma única doença, diabetes tipo 1, ao invés de um efeito gradativo relacionado à idade resultantes de duas doenças distintas. Ainda não há estudos gêmeos de LADA. Dos genes implicados na suscetibilidade genética ao diabetes tipo 1 e LADA, os mais importantes, para ambos, estão na região de histocompatibilidade [antígeno leucocitário humano (HLA)] do cromossomo 6 Campo et al. 2002).

O risco de diabetes tipo 1 está associado a genes na região MHC (que inclui o IDDM1 locus), em particular com uma frequência aumentada dos alelos HLA de classe II DR3 e / ou DR4 e DQ2 e / ou DQ8 em comparação com a população em geral. Estudos transversais mostraram que a idade no diagnóstico do diabetes tipo 1 clássico está inversamente relacionada à frequência dos fenótipos HLA de maior risco. Os fenótipos HLA de maior risco para diabetes tipo 1, DR3 / 4 e DQ2 / 8, foram mais prevalentes em pacientes LADA do que em indivíduos de controle saudáveis, consistentes com a conhecida predisposição genética à autoimunidade das ilhotas. No entanto, nenhuma diferença consistente nos alelos de classe IIDR3, DR4, DQ2 ou DQ8 foram identificados no LADA em comparação com o diabetes tipo 1 clássico. Um estudo comparou LADA com diabetes tipo 1 de início juvenil e descobriu que o de maior risco DQ2 / 8 o fenótipo não foi tão frequente em LADA [13], consistente com o aparecimento tardio de diabetes. Quando o LADA foi comparado com diabetes tipo 1 de início na idade adulta não houve diferenças consistentes na frequência dos alelos de classe II, sugerindo que eles têm uma base genética semelhante. A análise dos genes HLA classe II e classe I em todo o espectro do diabetes autoimune, abrangendo diabetes tipo 1 de início juvenil, diabetes tipo 1 de início na idade adulta e LADA em diferentes populações étnicas, iria informar ainda mais nossa compreensão da genética do diabetes autoimune que se apresenta em diferentes idades (Cerna et al. 2003)

O IDDM2 locus de suscetibilidade genética, que mapeia para um número variável de repetições em tandem (VNTR) a montante de INS (que codifica a pró-insulina humana) está associada ao diabetes tipo 1. Alelos VNTR classe I curtos 
predispõem ao diabetes tipo 1, enquanto alelos classe III mais longos são protetores. É a hipótese de que IDDM2 regula a expressão da pró-insulina no timo $\mathrm{e}$, portanto, a seleção de células $\mathrm{T}$ reativas à pró-insulina e a aquisição de tolerância imunológica à pró-insulina. A evidência de autoimunidade à (pró) insulina na forma de autoanticorpos não é uma característica do LADA (Cerrone et al. 2004). No entanto, pode ser informativo determinar a frequência de IDDM2 alelos em LADA em relação à população em geral e ao diabetes tipo 1. Dois estudos, de fato, implicaramIDDM2 em LADA. O alelo VNTR classe I foi mais frequente em adultos com diabetes com anticorpos GAD positivos. Em comparação com indivíduos de controle não diabéticos, o VNTR classe I era, como em indivíduos com diabetes tipo 1, mais frequente em indivíduos LADA, e o alelo de classe I mais curto também foi mais frequente nesses indivíduos. Essas descobertas aumentam a possibilidade de que os indivíduos LADA possam ser distinguidos por respostas aumentadas de células $\mathrm{T}$ à pró-insulina. Além disso, o gene para MHC de classe I relacionado à cadeia A (MICA) foi associado a LADA e diabetes tipo 1. Outros genes candidatos em LADA incluem aqueles para proteína 4 associada a linfócitos T citotóxicos e TNF-2 (Behanova et al. 2004).

Os alelos HLA associados à suscetibilidade ao diabetes tipo 1 incluem HLA DR3, DQB1 0201 e DR4, DQB1 * 0302, enquanto outros estão associados à proteção contra doenças, por exemplo HLADR2, DQB1 0602 (16-19). Crianças com diabetes tipo 1 mostram uma prevalência aumentada dos alelos heterozigotos HLADR3, DQB1 0201 eDR4, DQB1 0302, a proporção de heterozigotos diminuindo com a idade no diagnóstico. Crianças com o HLADR2 protetor de diabetes, DQB1 0602, têm pouca probabilidade de desenvolver diabetes, enquanto que no diabetes tipo 1 de início na idade adulta e LADA, os mesmos alelos têm menos proteção. No entanto, tanto o último quanto o LADA mostram susceptibilidade genética ao HLA com pouca ou nenhuma proteção genética ao HLA (Novota et al. 2004).

Surpreendentemente, mesmo adultos com diabetes que não requerem insulina, sem o autoanticorpo associado ao diabetes para GADA, têm um excesso de alelos HLA associados ao diabetes e são relativamente jovens e magros (Weets et al. 2002). Fatores genéticos relacionados à idade também influenciam o risco de diabetes tipo 1. Não só a incidência de diabetes tipo 1 por idade é menor em adultos do que em crianças, como também a faixa de incidência em todos os países europeus é reduzida em adultos. Além disso, existe um excesso de incidência masculina que se torna evidente durante a puberdade e é mais marcante na faixa etária de 25 a 29 anos Kyvik et al. 2004). Um estudo genético de Hosszufalusi et al. 2003, embora pequeno, encontrou genes de suscetibilidade HLA semelhantes em diabetes tipo 1 e LADA. Outros genes foram associados ao diabetes tipo 1 e esses genes, incluindo TNF, TNF, IL-10, polimorfismos do gene IL-6 e polimorfismo do promotor do gene IL-18, mas ainda precisam ser estudados de forma abrangente em LADA (Tsiavou et al. 2004). Outros polimorfismos genéticos nos genes CTLA4, PTPN22, IRS-1, ICOS e SUMO4 conferem um risco substancial para diabetes tipo 1 (Hornum et al. 2004). À luz dessas observações, ainda é possível que o LADA represente uma extremidade de um arco-íris de diabetes autoimune, que se distingue do diabetes tipo 1 clássico apenas porque é diagnosticado na idade adulta e apresenta algumas características clínicas, antropométricas e metabólicas mais comumente associada ao diabetes tipo 2.

O papel da resistência à insulina e sua contribuição para a fisiopatologia da LADA é controverso: o grau de resistência à insulina em LADA foi relatado como sendo menor do que no diabetes tipo 2 e comparável ao diabetes tipo 1 (Zinman et al. 2004). Recentemente, comparamos a resistência à insulina usando o modelo de homeostase em LADA, diabetes tipo 2 negativo para anticorpos e controles normais corrigindo o efeito do índice de massa corporal (IMC) (Chiu et al. 2007). Houve uma correlação positiva do IMC com a resistência à insulina em ambos LADA e diabetes tipo 2, e a resistência à insulina foi notavelmente semelhante em ambos os grupos quando corrigida para o IMC. Além disso, os indivíduos com LADA e diabetes tipo 2 eram mais resistentes à insulina do que os controles normais quando corrigidos para o IMC.

Alguns estudos relataram um IMC médio significativamente menor em LADA em comparação com pacientes com diabetes tipo 2, enquanto outros estudos não mostram uma diferença. No entanto, o intervalo de IMC é frequentemente grande, com tremendas sobreposições entre LADA e diabetes tipo 2. Um estudo recente em pacientes europeus adultos com diabetes 
mostrou que a prevalência da síndrome metabólica é significativamente maior em pacientes com diabetes tipo 2 do que em pacientes internados com LADA ou adultos com diabetes tipo 1; foi ainda demonstrado que a síndrome metabólica não é mais prevalente em pacientes internados com diabetes autoimune do que em indivíduos sem controle, e a síndrome metabólica não é uma característica do diabetes autoimune (Hawa et al. 2009).

\section{- Critérios diagnósticos}

O diagnóstico de LADA é atualmente baseado em três critérios: (1) idade adulta no início do diabetes; (2) a presença de autoanticorpos de ilhotas circulantes; e (3) falta de necessidade de insulina por pelo menos 6 meses após o diagnóstico. Os autoanticorpos das ilhotas são marcadores da autoimunidade das células beta pancreáticas que distinguem o LADA do diabetes tipo 2. Um período de independência da insulina após o diagnóstico tem como objetivo distinguir o LADA do diabetes tipo 1 clássico (Fourlanos et al. 2005). O limite mínimo de idade para LADA varia de 25 a 40 anos, mas é arbitrário. Idealmente, um corte de idade seria baseado em estudos longitudinais da história natural pré-clínica do LADA, usando os marcadores metabólicos e imunogenéticos que distinguem o LADA do diabetes tipo 1 clássico de início juvenil. Nesse ínterim, proporíamos que a idade mínima operacional fosse 30 anos (Cosentino et al. 2002).

O diagnóstico de LADA baseia-se principalmente na soropositividade de anticorpos para GAD; autoanticorpos de insulina (IAA) e anticorpos para o antígeno de insulinoma tipo tirosina fosfatase 2 (IA2) foram relatados como infrequentes em LADA. No entanto, um perfil preciso da imunidade humoral em LADA requer mais estudos para documentar a prevalência de IAA, anticorpos IA2 e ICA em LADA em diferentes populações. Anticorpos GAD não são condição sine qua non-adultos com diabetes que não são insulino-dependentes no momento do diagnóstico e têm qualquer autoanticorpo de ilhotas seriam atualmente classificados como portadores de LADA (Takeda et al. 2002).

Outro critério para LADA é um período mínimo de 6 meses de independência da insulina após o diagnóstico. Pelo menos três fatores influenciam o período de independência da insulina: (1) a história natural da doença; (2) o momento do diagnóstico em relação à história natural; e (3) o viés terapêutico do médico assistente. Isso vai variar de paciente para paciente. Indivíduos assintomáticos com diagnóstico de diabetes com base apenas no aumento da glicose no sangue têm maior probabilidade de atender ao critério de independência da insulina por um período mínimo do que aqueles diagnosticados com diabetes após se tornarem sintomáticos. A atual classificação diagnóstica é, portanto, tendenciosa, frequentemente excluindo pacientes sintomáticos e / ou com diagnóstico tardio de diabetes. Alguém com diabetes assintomático não diagnosticado por muitos meses, que eventualmente apresenta sintomas, provavelmente receberá injeções de insulina imediatamente e, portanto, será considerado portador de diabetes tipo 1 clássico. Se diagnosticada com diabetes mais cedo com base apenas na glicose no sangue, essa pessoa seria inicialmente independente da insulina e, se fosse positiva para anticorpos das ilhotas, provavelmente seria classificada como tendo LADA, e não diabetes tipo 1 clássico Fourlanos et al. 2005).

Além disso, a decisão de tratar pacientes LADA com agentes orais ou insulina reflete também o julgamento do médico assistente. Alguns pacientes com deficiência acentuada de insulina serão tratados com hipoglicemiantes orais, quando deveriam ter sido tratados com insulina desde o início. Por outro lado, alguns pacientes com produção endógena adequada de insulina serão tratados com insulina desde o início, quando poderiam ter alcançado o controle glicêmico adequado com agentes orais. Assim, um fator preponderante é se o médico assistente é ou não pró-ativo no que diz respeito ao tratamento com insulina. Claramente, um maior conhecimento da história natural e patogênese do LADA é necessário para refinar os critérios diagnósticos objetivos. um fator primordial é se o médico assistente é ou não pró-ativo no que diz respeito ao tratamento com insulina. Claramente, é necessário um maior conhecimento da história natural e patogênese do LADA para refinar os critérios diagnósticos objetivos. um fator primordial é se o médico assistente é ou não pró-ativo no que diz respeito ao tratamento com 
insulina. Claramente, um maior conhecimento da história natural e patogênese do LADA é necessário para refinar os critérios diagnósticos objetivos Fourlanos et al. 2005).

Antes da introdução de testes de autoanticorpos de ilhotas, estudos mostraram que o nível de secreção de peptídeo C ajudou a prever a necessidade de insulina em adultos com diabetes, sugerindo que a independência da insulina na prática clínica seja corroborada pela demonstração formal da produção adequada de insulina no momento do diagnóstico. A insulina endógena ou o peptídeo C podem ser medidos em jejum e após estimulação com glicose oral, uma refeição mista, glucagon intravenoso ou outros secretagogos, como arginina intravenosa. No entanto, a sensibilidade comparativa desses estímulos e as faixas de referência da população saudável padronizadas para idade e índices antropométricos ainda precisam ser estabelecidas. $\mathrm{O}$ objetivo deve ser demonstrar produção de insulina endógena satisfatória antes de continuar com agentes hipoglicemiantes orais e modificação do estilo de vida, para atingir glicemia satisfatória e IMC normal $(<25,0 \mathrm{~kg} / \mathrm{m} 2)$ não deve ser um critério de diagnóstico para LADA (Ramachandra et al. 2009).

\section{- Associação com doenças autoimunes}

A associação de outras doenças autoimunes com diabetes tipo 1, especialmente doença autoimune da tireoide e doença celíaca, está bem estabelecido. O agrupamento dessas doenças autoimunes está relacionado aos haplótipos de risco comuns HLA DR3-DQ2, DR4-DQ8 (Mayer et al. 2007) Antes de LADA ser formalmente definido, uma forma lentamente progressiva de diabetes tipo 1 foi associada a outras doenças autoimunes específicas de órgãos e vista como parte da síndrome polendócrina autoimune tipo 2 [40] Em crianças com diabetes tipo 1, a prevalência relatada de tireoidite autoimune é de 4\% e de anticorpos tireoidianos associados (anticorpos peroxidase tireoidiana [TPO], anticorpos tireoglobulina [TG]) $16 \%$. Aproximadamente $25 \%$ dos adultos com diabetes autoimune apresentam evidência sorológica de tireoidite autoimune. Um grande estudo italiano encontrou uma alta frequência de anticorpos TPO em pacientes LADA (24\%) em comparação com pacientes com diabetes tipo 2 (5\%). Não há relatórios sobre a frequência de doenças clínicas da tireoide em LADA. A doença celíaca ocorre em aproximadamente $10 \%$ das crianças com diabetes tipo 1. Uma alta frequência (19\%) de anticorpos para gliadina foi relatada no LADA, mas a prevalência da doença celíaca confirmada por biópsia é desconhecida. Um aumento da frequência de anticorpos para 21-hidroxilase e 17-hidroxilase, marcadores de hipoadrenalismo autoimune (Addison's), também foi relatado em LADA (Borg et al. 2002).

\section{Conclusão}

Em conclusão, um dos grandes desafios que a ciência clínica enfrenta é a categorização precisa dos subtipos dentro das entidades de doenças, de modo que possam eventualmente ser combinados a exposições genéticas e ambientais específicas. Assim, o DM autoimune é altamente prevalente em adultos. Não se sabe se o mecanismo de destruição das células beta é igual ao dos jovens e se irão responder de forma semelhante à terapia imunomoduladora ou imunossupressora. Não há, ainda, recomendação para dosar autoanticorpos em adultos com DM2. Diagnosticar o diabetes autoimune em indivíduos erroneamente classificados como tipo 2 é importante, pois alerta para provável deficiência de insulina, evitando o atraso na insulinoterapia. A frequente associação entre LADA e autoimunidade tiroidiana também reforça esta avaliação nesses pacientes. Desse modo, é de suma importância que pesquisas futuras abordem esta temática, a fim de melhor elucidação da fisiopatologia do LADA, bem como suas manifestações clínicas, a fim de melhor propedêutica a esses pacientes.

\section{Referências}

Behanova, M. M., Vankova, M. \& Hill, M. (2004). O polimorfismo de INS VNTR está associado a anticorpos descarboxilase do ácido glutâmico e peptídeo C pós-prandial em pacientes com início de diabetes após os 35 anos de idade. Physiol Res., 53 (10), 187-190. 
Borg, H., Gottsäter, A., Fernlund, P. \& Sundkvist, G. (2002) 12 anos estudo prospectivo da relação entre os anticorpos das ilhotas e função celular durante e após o diagnóstico em pacientes com diabetes no início da vida adulta. Diabetes, 51 (12), 1754-1762.

Calsolari, M. R., Rosário, P. W. S., Reis, J. S., Silva, S. C. \& Purisch, S. (2008). Diabetes autoimune latente do adulto ou diabetes melito tipo 2 magro? Arq. Bras. Encodrinol. Metab, 52 (2), 1-13.

Campo, L. L. (2002). Estudos de ligação e associação genética de diabetes tipo 1: desafios e recompensas. Diabetologia, 45 (1), 21-35

Cerna, M., Novota, P. \& Kolostova, K. (2003). HLA em pacientes adultos tchecos com diabetes mellitus autoimune: comparação com crianças checas com diabetes tipo 1 e pacientes com diabetes tipo 2. Eur J Immunogenet, 30 (11), 401-407

Cerrone, G. E., Caputo, M. \& Lopez, A. P. (2004). O número variável de repetições em tandem do gene da insulina determina a suscetibilidade ao diabetes autoimune latente em adultos. Mol Diagn, 8 (2), 43-49

Chiu, H. K., Tsai, E. C., Stoever, J., Worrel, B. B., Goel. A. \& Palmer, J. P. (2007). Resistência à insulina equivalente em diabetes autoimune latente em adultos (LADA) e pacientes com diabetes tipo 2. Diabetes Res Clin Pract, 77 (9), 237-244

Cosentino, A., Gambelunghe, G., Tortoioli, C. \& Falorni, A. (2002). O polimorfismo do gene CTLA-4 contribui para o risco genético de diabetes autoimune latente em adultos. Ann NY Acad Sci, 958 (5), 337-440

Couri, C. E., Oliveira, M. C. B., Stracieri, A. B. P. L., Moraes, D. A., Pieroni, F., Barros, G. M. N. \& Madeira, M. I. A. (2009). C-peptide levels and insulin independence following autologous nonmyeloablative hematopoietic stem cell transplantation in newly diagnosed type 1 diabetes mellitus. JAMA, 301 (15), $1573-1579$

Fourlanos, S., Dotta, F., Greenbaum, C. J., Palmer, J. P., Rolandsson, O., Colman, P. G. \& Harrison, L. C. (2005). Diabetes autoimune latente em adultos (LADA) deve ser menos latente. Diabetologia, 48 (1), 2206-2212

Hawa, M. I., Thivolet, C., Mauricio, D., Alemanno, I., Cipponeri, E., Collier, D., Hunter, S., Buzzetti, R., de Leiva, A., Pozzilli, P. \& Leslie, R. D. (2009). Síndrome metabólica e diabetes autoimunológico. Diabetes Care, 32 (7), 160-164

Hornum, L. \& Markholst, H. (2004). Novos genes autoimunes e a patogênese do diabetes tipo 1. Curr Diabet Rep., 4 (9), 135-142

Hosszufalusi, N., Vatay, A., Rajczy, K., Prohaszka, Z., Pozsonyi, E., Horvath, L., Grosz, A., Gero, L., Madacsy, L., Romics, L., Karadi, I., Fust, G. \& Panczel, P. (2003). Características genéticas semelhantes e diferentes padrões de autoanticorpos de células ilhotas de diabetes autoimune latente em adultos (LADA) em comparação com diabetes tipo 1 de início na idade adulta com rápida progressão. Diabetes Care, 26 (8), 452-457

Hyttinen, V., Kaprio, J., Kinnunen, L., Koskenvuo, M. \& Tuomilehto, J. (2003). Responsabilidade genética do diabetes tipo 1 e a idade de início entre 22.650 jovens pares de gêmeos finlandeses: um estudo de acompanhamento nacional. Diabetes, 52 (2), 1052-1055

Jones A. G., McDonald, T. J., Shields, B. M,m Hagopian, W. \& Hattersley, A. T. (2021). Latent Autoimmune Diabetes of Adults (LADA) is likely to representant a mixed population of autoimmune (type 1) and nonautoimmune (type 2) diabetes. Diabetes Care, 44 (1), 1-9

Kyvik, K. O., Nystrom, L., Gorus, F., Songini, M., Oestman, J., Castell, C., Green, A., Guyrus, E., Ionescu-Tirgoviste, C., Mckinney, P. A., Michalkova, D., Ostrauskas, R. \& Raymond, N. T. (2004). A epidemiologia do diabetes mellitus tipo 1 não é a mesma em adultos jovens e em crianças. Diabetologia, 47 (3), $377-384$

Leslie, R.D. G., Williams, R. \& Pozzilli, P. (2006). Diabetes tipo 1 e diabetes autoimune latente em adultos: uma ponta do arco-íris. The Journal of Clinical Endocrinology \& Metabolism, 91 (5), 1654-1659

Liao, Y., Xiang, Y. \& Zhou, Z. (2012). Diagnostic criteria of latent autoimmune diabetes in adults (LADA): a review and reflection. Front Med. 6 (3), $243-247$

Mayer, A., Fabien, N., Gutowski, M. C., Dubois, V., Gebuhrer, L., Bienvenu, J., Orgiazzi, J. \& Madec, A. M. (2007). Autoimunidade celular e humoral contrastante associada a adultos com diabetes autoimunitário latente. Eur J Endocrinol, 157 (10), 53-61

Novota, P., Kolostova, K., Pinterova, D., Novak, J., Treslova, L., Andel, M. \& Cerna, M. (2005). Polimorfismos do promotor do gene da interleucina-18 em pacientes adultos com diabetes mellitus tipo 1 e diabetes autoimune latente em adultos. Immunol Lett., 96 (3), 247-251

Ramachandra, G., Naik, B. M., Brooks-Worrell \& Palmer, J. P. (2009). Diabetes autoimune latente em adultos. J Clin Endocrinol Metab. 94 (12), $4635-4644$ Silva, M. E. R. (2007). Precisamos diagnosticar o diabetes Latente Autoimune do Adulto (LADA)? Arq. Bras. Encodrinol. Metab, 51 (1), 1-8.

Takeda, H., Kawasaki, E. \& Shimizu, I. (2002). Clinical, autoimmune, and genetic tools of adult-early diabetic patients with GAD Autoanticorpos in Japan (Ehime Study). Diabetes Care 25 (6), 995-1001

Tsiavou, A., Hatziagelaki, E., Chaidaroglou, A., Manginas, A., Koniavitou, K., Degiannis, D. \& Raptis, S. A. (2004). TNF-, TGF-1, IL-10, IL-6, polimorfismos genéticos em diabetes autoimune latente de adultos (LADA) e diabetes mellitus tipo 2. J Clin Immunol, 26 (4), 591-599

Weets, I., Siraux, V., Daubresse, J. C., De Leeuw, I. H., Fery, F., Keymeulen, B., Krzentowski, G., Letiexhe, M., Mathieu, C., Nobels, F., Rottiers, R., Scheen, A., Van Gaal, L., Schuit, F. C., Van der Auwera, B., Rui, M., De Pauw, P., Kaufman, L. \& Gorus, F. K. (2002). Belgian Diabetes Registry: relação entre o fenótipo da doença e o genótipo HLA-DQ em pacientes diabéticos diagnosticados no início da idade adulta. J Clin Endocrinol Metab., 87 (4), $2597-2605$

Zinman, B., Kahn, S. E., Haffner, S. M., O'Neill, M. C., Heise, M. A. \& Freed, M. I. (2004). Características fenotípicas de pacientes com diabetes tipo 2 recentemente diagnosticados com anticorpos GAD positivos na América do Norte e Europa. Diabetes, 53 (8), 3193-3200 